\title{
Generalized Heteroskedasticity ACF for Moving Average Models in Explicit Forms
}

\author{
Samir K. Safi \\ Associate Professor of Statistics \\ Department of Economics and Statistics \\ The Islamic University of Gaza \\ Gaza, Palestine \\ samirsafi@gmail.com
}

\begin{abstract}
The autocorrelation function $(\mathrm{ACF})$ measures the correlation between observations at different distances apart. We derive explicit equations for generalized heteroskedasticity ACF for moving average of order $q$, $M A(q)$. We consider two cases: Firstly: when the disturbance term follow the general covariance matrix structure $\operatorname{Cov}\left(w_{i}, w_{j}\right)=\Sigma$ with $\sigma_{i, j} \neq 0 \forall i \neq j$. Secondly: when the diagonal elements of $\Sigma$ are not all identical but $\sigma_{\mathrm{i}, \mathrm{j}}=0 \forall \mathrm{i} \neq \mathrm{j}$, i.e. $\Sigma=\operatorname{diag}\left(\sigma_{11}, \sigma_{22}, \ldots, \sigma_{\mathrm{tt}}\right)$. The forms of the explicit equations depend essentially on the moving average coefficients and covariance structure of the disturbance terms.
\end{abstract}

Keywords: Heteroscedasticity; Homoscedasticity; Autocorrelation; Moving Average; Covariance.

\section{Introduction}

In time-series and regression models, basic forms of models make use of the assumption that the disturbances $w_{i}$ have the same variance across all observation points. When this assumption is violated, the disturbances have heteroscedasticity, and this behavior will be reflected in the disturbances estimated from a fitted model.

Heteroskedasticity naturally occurs when the constant variance of the disturbance term is violated. The constant variance of the disturbance means $\operatorname{Var}\left(w_{t}\right)=\sigma^{2}, t=1,2, \ldots$ With heteroskedasticity, this disturbance term variance is not constant. Instead, the variance of the distribution of the disturbance term depends on exactly which observation is being discussed, $\operatorname{Var}\left(w_{t}\right)=\sigma_{t}^{2}, t=1,2, \ldots$ The most frequently specified model of heteroskedasticity relates the variance of the disturbance term to an exogenous variable such as $Z_{i}$, for example $\operatorname{Var}\left(w_{t}\right)=\sigma^{2} Z_{t}^{2}, t=1,2, \ldots$ where $Z$, the "proportionality factor," may or may not be in the model, Studenmund (2011).

In the case of heteroskedastic residual, the covariance matrix estimator is not consistent for the true estimator covariance, so that the most serious implications of heteroskedastic residual is not the resulting inefficiency of ACF but the misleading inference when standard tests are used. 
The heteroskedasticity of the residuals exists, whenever the covariance matrix of the residuals is not a diagonal matrix, i.e. whenever $\operatorname{Cov}\left(w_{i}, w_{j}\right)=\Omega$ with $\sigma_{i, j} \neq 0$ for some $i \neq j$.

A moving-average process is a time-series process in which the current value of a variable is modeled as a weighted average of current and past realizations of a white noise process and, optionally, a time-invariant constant. By convention, the weight on the current realization of the white-noise process is equal to one, and the weights on the past realizations are known as the moving-average $(M A)$ coefficients.

The regular autocorrelation function (ACF) produces spurious results when we do not account for heteroskedasticity of the residuals. In case the variance is proportional to the level of the series, a logarithmic transformation may make the series both homoscedastic and stationary in variance. But many time series do not have constant, or even stationary variance even after transformations, Stockhammar (2010).

The ACF plays a crucial role in studying the correlation structure of weakly stationary time series, Dong et al. (2012). It is well known that for a causal $M A(q)$ model, its ACF "cuts off" after lag $q$; that is, it is zero, (Cryer and Chan 2008).

The basic ACF is modeled under an assumption of constant variance. This phenomena is known as homoscedasticity. If they are not, it causes serious problems for our estimates and must be corrected in order to obtain reliable estimates. The exact formula for ACF for $M A(q)$ models can be obtained although its closed form when the disturbance terms are identically distributed, i.e. they have the same variance for all observations . However, for a general covariance structure of the disturbance terms, it is rather difficult to obtain an exact formula for the ACF.

There is a large literature on estimating ACF in the presence of heteroscedasticity, see for example, Bera, A. et al. (2005), and Wallentin and Agren (2002). Studies of many econometric time series models for financial markets revealed that it is unreasonable to assume that conditional variance of the disturbance term is constant, as it for many stochastic processes. Various procedures are available to test for the possibility that the disturturbance terms of a linear regression model are autocorrelated in a first order process with a constant autoregressive coefficient. (See for example Bumb and Kelejian, 1983).

Demos (2000) discussed the statistical properties of conditionally heteroskedastic in mean models. He derived expressions for the autocovariance of the observed series under the assumption that the conditional variance follows a flexible parameterization.

Safi $(2009,2011)$ derived explicit equations for ACF in the presence of heteroscedasticity disturbances in first order autoregressive, $A R(1)$, and $p$-th order autoregressive, $A R(p)$, model. He showed two cases: (1) when the disturbance follows the general covariance matrix, $\Sigma$, and (2) when the diagonal elements of $\Sigma$ are not all identical but $\sigma_{\mathrm{i}, \mathrm{j}}=0 \forall \mathrm{i} \neq \mathrm{j}$, i.e. $\Sigma=\operatorname{diag}\left(\sigma_{11}, \sigma_{22}, \ldots, \sigma_{\mathrm{tt}}\right)$. 
Praetz (2008) discussed the effect of auto-correlated disturbances when they are not modeled on the statistics used in drawing inferences in the multiple linear regression model. He derived biases for the $\mathrm{F}$ and $\mathrm{R}^{2}$ statistics and evaluates them numerically for an example. He discussed the reflections for empirical research on the causes, detection and treatment of autocorrelation.

In this paper, for a general covariance structure of the disturbance terms, we investigate the ACF of heteroskedastic in the residuals for an $M A(q)$ by employing a general formulation for $M A$ models. In particular, we derive explicit equations for General Heteroscedastic Autocorrelation Function (GHACF) for the general moving average of order $q, M A(q)$. We consider two cases: firstly when the disturbance terms follow the general covariance matrix structure $\operatorname{Cov}\left(w_{i}, w_{j}\right)=\Sigma$ with $\sigma_{i, j} \neq 0$ and all $i \neq j$ and secondly when the diagonal elements of $\Sigma$ are not all identical but $\sigma_{i, j}=0 \forall i \neq j$, i.e. $\Sigma=\operatorname{diag}\left(\sigma_{11}, \sigma_{22}, \ldots, \sigma_{\mathrm{tt}}\right)$. The forms of the explicit equations depend essentially on the moving average coefficients and covariance structure of the disturbance terms.

The rest of this paper is structured as follows. In section 2 we derive explicit equations for $\mathrm{GHACF}$ for an $M A(q)$. Explicit equations for Heteroscedastic Autocorrelation Function (HACF) for an $M A(q)$ are described in section 3. Section 4 summarizes the results and offers suggestions for future research on deriving explicit equations for ACF in the presence of heteroscedasticity disturbances for different time series mixed Autoregressive Moving Average Model (ARMA).

\section{General Heteroscedastic Autocorrelation Function (GHACF)}

Suppose $Z_{t}$ is linearly dependent on a weighted finite number $q$ of previous shocks or noise $w_{t}$ such that

$$
Z_{t}=\sum_{j=0}^{\infty} \psi_{j} w_{t-j},
$$

where $\psi_{0}=1, Z_{t}=Z_{t}-\mu$ is the deviation of the model from some origin, or from its mean, if the model is stationary. Such a sequence of random variables $w_{t}, w_{t-1}, w_{t-2}, \ldots$ is called a white noise model.

Definition 2.1. Consider the special case of (2.1) when only the first $q$ of the $\psi$ weights are nonzero, and without loss of generality, assume $\mu=0$. The moving average model of order $q$, or $M A(q)$ model, is defined to be (See for example, Shumway and Stoffer 2011)

$$
Z_{t}=\sum_{j=0}^{q} \theta_{j} w_{t-j},
$$

where $\mathrm{Z}_{\mathrm{t}}$ is the time series under investigation, $\theta_{0}=1$, and $w_{t}$ is a Gaussian white noise series with mean zero and variance $\sigma_{w}^{2}$. There are $q$ lags in the moving average and 
$\theta_{1}, \theta_{2}, \ldots, \theta_{q}\left(\theta_{q} \neq 0\right)$ are parameters. The $M A(q)$ model may be written in terms of backward shift operator $B$.

$$
Z_{t}=\theta(B) w_{t}
$$

where $\theta(B)=1+\theta_{1} B+\theta_{2} B^{2}+\cdots+\theta_{q} B^{q}$ is called the moving average operator with $B^{j} w_{t}=w_{t-j}$

For GHACF, we assume that the white noise term has mean zero, $E\left(w_{t}\right)=0$, and the covariance matrix $\operatorname{Cov}\left(w_{i}, w_{j}\right)=\Sigma$ where

$$
\Sigma=\left[\begin{array}{cccc}
\sigma_{11} & \sigma_{12} & \ldots & \sigma_{1 t} \\
\sigma_{21} & \sigma_{22} & \ldots & \sigma_{2 t} \\
\vdots & \vdots & \ddots & \vdots \\
\sigma_{t 1} & \sigma_{t 2} & \ldots & \sigma_{t t}
\end{array}\right]
$$

Definition 2.2. The covariance between $Z_{t}$ and $Z_{t+h}$, separated by $h$ intervals of time (which under the stationary assumption must be the same for all $t$ ) is called the autocovariance function at $\operatorname{lag} h(A C V F)$ and is defined by

$$
\gamma(h)=\operatorname{Cov}\left(Z_{t+h}, Z_{t}\right)=E\left[\left(Z_{t+h}-\mu\right)\left(Z_{t}-\mu\right)\right]
$$

In this paper we are assuming that $Z_{t}$ has zero mean. We can always introduce a nonzero mean by replacing $Z_{t}$ by $Z_{t-\mu}$ throughout our equations.

Definition 2.3. The autocorrelation function at lag $h(A C F)$, that is the correlation between $Z_{t}$ and $Z_{t+h}$ is defined by

$$
\rho(h)=\frac{\gamma(t+h, t)}{\sqrt{\gamma(t+h, t+h) \gamma(t, t)}}=\frac{\gamma(h)}{\gamma(0)},
$$

where $\gamma_{0}=\sigma_{Z}^{2}$ is the same at time $t+h$ as at time $t$.

The Cauchy-Schwarz inequality shows that $-1 \leq \rho(h) \leq 1$ for all $h$, enabling one to assess the relative importance of a given autocorrelation value by comparing with the extreme values -1 and 1 . 
Theorem 2.4. Consider the general $M A(q)$ model, $Z_{t}=\sum_{j=0}^{q} \theta_{j} w_{t-j}$, with $E\left(w_{t}\right)=0$ and $\operatorname{Cov}\left(w_{i}, w_{j}\right)=\Sigma$, where $\Sigma$ is given in (2.4) and $\sigma_{i, j} \neq 0 \forall i \neq j$. Then the GHACF at lag $h$ is given by

$$
\rho(h)= \begin{cases}\frac{\sum_{j=0}^{q} \sum_{i=0}^{q} \theta_{i} \theta_{j} \sigma_{t-i, t-h-j}}{\sum_{j=0}^{q} \sum_{i=0}^{q} \theta_{i} \theta_{j} \sigma_{t-i, t-j}} & 1 \leq h \leq q \\ 0, & h>q\end{cases}
$$

Proof: Using (2.2) and (2.5), the ACVF at lag $h$ for $h=1,2, \ldots, q$ is

$$
\begin{aligned}
\gamma(h) & =E\left(Z_{t} Z_{t-h}\right) \\
& =E\left[\left(w_{t}+\theta_{1} w_{t-1}+\cdots+\theta_{q} w_{t-q}\right)\left(w_{t-h}+\theta_{1} w_{t-h-1}+\cdots+\theta_{q} w_{t-h-q}\right)\right] \\
& =E\left(w_{t} w_{t-h}\right)+\theta_{1} E\left(w_{t} w_{t-h-1}\right)+\cdots+E\left(\theta_{q} w_{t} w_{t-h-q}\right) \\
& +E\left(\theta_{1} w_{t-1} w_{t-h}\right)+\theta_{1}^{2} E\left(w_{t-1} w_{t-h-1}\right)+\cdots+\theta_{1} \theta_{q} E\left(w_{t-1} \theta_{q} w_{t-h-q}\right) \\
& +\theta_{2} E\left(w_{t-2} w_{t-h}\right)+\theta_{2} \theta_{1} E\left(w_{t-2} w_{t-h-1}\right)+\cdots+\theta_{2} \theta_{q} E\left(w_{t-2} w_{t-h-q}\right) \\
& \vdots \\
& +\theta_{q} E\left(w_{t-q} w_{t-h}\right)+\theta_{q} \theta_{1} E\left(w_{t-q} w_{t-h-1}\right)+\cdots+\theta_{q}^{2} E\left(w_{t-q} w_{t-h-q}\right)
\end{aligned}
$$

Collecting terms, we find that the ACVF for $M A(q)$ at $\operatorname{lag} h$ is

$$
\gamma(h)= \begin{cases}\sum_{j=0}^{q} \sum_{i=0}^{q} \theta_{i} \theta_{j} \sigma_{t-i, t-h-j}, & 0 \leq h \leq q \\ 0, & h>q\end{cases}
$$

Using (2.8), the ACVF for an $M A(q)$ at lag 0, i.e. the variance of an $M A(q)$ model is

$$
\gamma(0)=\sum_{j=0}^{q} \sum_{i=0}^{q} \theta_{i} \theta_{j} \sigma_{t-i, t-j}
$$

Dividing (2.9) by (2.10), yields (2.7), and that completes the proof. 


\section{Special Cases}

The next corollaries (2.5) and (2.6) derive the GHACF at lag $h$ for an $M A(1)$ and $M A(2)$ models.

Corollary 2.5. Consider $M A(1)$ model, $Z_{t}=w_{t}+\theta w_{t-1}$, with $E\left(w_{t}\right)=0$ and $\operatorname{Cov}\left(w_{i}, w_{j}\right)=\Sigma$, where $\Sigma$ is given in (2.4) and $\sigma_{i, j} \neq 0 \forall i \neq j$. Then the GHACF for $M A(1)$ at $\operatorname{lag} h$ is given by

$$
\rho(h)= \begin{cases}\frac{\sigma_{t, t-1}+\theta\left(\sigma_{t, t-2}+\sigma_{t-1, t-1}\right)+\theta^{2} \sigma_{t-1, t-2}}{\sigma_{t, t}+2 \theta \sigma_{t, t-1}+\theta^{2} \sigma_{t-1, t-1}}, & h=1 \\ 0, & h \geq 2\end{cases}
$$

Proof: Using (2.8) we obtain the variance of for an $M A(1)$ model,

$$
\begin{aligned}
\gamma(0) & =E\left(Z_{t}^{2}\right) \\
& =E\left[\left(w_{t}+\theta w_{t-1}\right)\left(w_{t}+\theta w_{t-1}\right)\right] \\
& =E\left(w_{t}^{2}\right)+\theta E\left(w_{t} w_{t-1}\right)+E\left(\theta w_{t-1} w_{t}\right)+\theta^{2} E\left(w_{t-1}^{2}\right)
\end{aligned}
$$

Then,

$$
\gamma(0)=\sigma_{t, t}+2 \theta \sigma_{t, t-1}+\theta^{2} \sigma_{t-1, t-1}
$$

Using (2.8), the ACVF for an MA(1) at lag 1 is

$$
\begin{aligned}
\gamma(1) & =E\left(Z_{t} Z_{t-1}\right) \\
& =E\left[\left(w_{t}+\theta w_{t-1}\right)\left(w_{t-1}+\theta w_{t-2}\right)\right] \\
& =E\left(w_{t} w_{t-1}\right)+\theta E\left(w_{t} w_{t-2}\right)+E\left(\theta w_{t-1} w_{t-1}\right)+\theta^{2} E\left(w_{t-1} w_{t-2}\right)
\end{aligned}
$$

Then,

$$
\gamma(1)=\sigma_{t, t-1}+\theta\left(\sigma_{t, t-2}+\sigma_{t-1, t-1}\right)+\theta^{2} \sigma_{t-1, t-2}
$$

Similarly, $\gamma(h)=0$ for $h \geq 2$.

Dividing (2.13) by (2.12), yields (2.11), and that completes the proof. 
Corollary 2.6. Consider $M A(2)$ model, $Z_{t}=w_{t}+\theta_{1} w_{t-1}+\theta_{2} w_{t-2}$, with $E\left(w_{t}\right)=0$ and $\operatorname{Cov}\left(w_{i}, w_{j}\right)=\Sigma$, where $\Sigma$ is given in (2.4) and $\sigma_{i, j} \neq 0 \forall i \neq j$. Then the GHACF for an $M A(2)$ at lag $h$ is given by

$$
\begin{gathered}
\rho(1)=\frac{\left[\begin{array}{c}
\sigma_{t, t-1}+\theta_{1}\left(\sigma_{t, t-2}+\sigma_{t-1, t-1}\right)+\theta_{2}\left(\sigma_{t, t-3}+\sigma_{t-2, t-1}\right) \\
+\theta_{1}^{2} \sigma_{t-1, t-2}+\theta_{2}^{2} \sigma_{t-2, t-3}+\theta_{1} \theta_{2}\left(\sigma_{t-1, t-3}+\sigma_{t-2, t-2}\right)
\end{array}\right]}{\left[\begin{array}{l}
\sigma_{t, t}+\theta_{1} \sigma_{t, t-1}+\theta_{2} \sigma_{t, t-2}+\theta_{1} \sigma_{t-1, t}+\theta_{1}^{2} \sigma_{t-1, t-1} \\
+\theta_{1} \theta_{2} \sigma_{t-1, t-2}+\theta_{2} \sigma_{t-2, t}+\theta_{2} \theta_{1} \sigma_{t-2, t-1}+\theta_{2}^{2} \sigma_{t-2, t-2}
\end{array}\right]} \\
\rho(2)=\frac{\left[\begin{array}{l}
\sigma_{t, t-2}+\theta_{1}\left(\sigma_{t, t-3}+\sigma_{t-1, t-2}\right)+\theta_{2}\left(\sigma_{t, t-4}+\sigma_{t-2, t-2}\right) \\
+\theta_{1}^{2} \sigma_{t-1, t-3}+\theta_{2}^{2} \sigma_{t-2, t-4}+\theta_{1} \theta_{2} \sigma_{t-1, t-4}+\theta_{2} \theta_{1} \sigma_{t-2, t-3}
\end{array}\right]}{\left[\begin{array}{c}
\sigma_{t, t}+\theta_{1} \sigma_{t, t-1}+\theta_{2} \sigma_{t, t-2}+\theta_{1} \sigma_{t-1, t}+\theta_{1}^{2} \sigma_{t-1, t-1} \\
+\theta_{1} \theta_{2} \sigma_{t-1, t-2}+\theta_{2} \sigma_{t-2, t}+\theta_{2} \theta_{1} \sigma_{t-2, t-1}+\theta_{2}^{2} \sigma_{t-2, t-2}
\end{array}\right]} \\
\rho(h)=0 \text { for h } \mathrm{h} 3
\end{gathered}
$$

Proof: Using (2.8) we obtain the variance for an $M A(2)$ model,

$$
\begin{aligned}
\gamma(0) & =E\left(Z_{t}^{2}\right) \\
& =E\left(w_{t}^{2}\right)+\theta_{1} E\left(w_{t} w_{t-1}\right)+E\left(\theta_{2} w_{t} w_{t-2}\right)+E\left(\theta_{1} w_{t-1} w_{t}\right)+\theta_{1}^{2} E\left(w_{t-1}^{2}\right) \\
& +\theta_{1} \theta_{2} E\left(w_{t-1} w_{t-2}\right)+\theta_{2} E\left(w_{t-2} w_{t}\right)+\theta_{2} \theta_{1} E\left(w_{t-2} w_{t-h-1}\right)+\theta_{2}^{2} E\left(w_{t-2}^{2}\right)
\end{aligned}
$$

Collecting terms, then the variance for an $M A(2)$ model is

$$
\begin{aligned}
\gamma(0) & =\sigma_{t, t}+\theta_{1} \sigma_{t, t-1}+\theta_{2} \sigma_{t, t-2}+\theta_{1} \sigma_{t-1, t}+\theta_{1}^{2} \sigma_{t-1, t-1} \\
& +\theta_{1} \theta_{2} \sigma_{t-1, t-2}+\theta_{2} \sigma_{t-2, t}+\theta_{2} \theta_{1} \sigma_{t-2, t-1}+\theta_{2}^{2} \sigma_{t-2, t-2}
\end{aligned}
$$

Using (2.8), the ACVF for an $M A(2)$ at $\operatorname{lag} 1$ is

$$
\begin{aligned}
\gamma(1) & =E\left(Z_{t} Z_{t-1}\right) \\
& =E\left(w_{t} w_{t-1}\right)+\theta_{1} E\left(w_{t} w_{t-2}\right)+E\left(\theta_{2} w_{t} w_{t-3}\right)+E\left(\theta_{1} w_{t-1} w_{t-1}\right)+\theta_{1}^{2} E\left(w_{t-1} w_{t-2}\right) \\
& +\theta_{1} \theta_{2} E\left(w_{t-1} w_{t-3}\right)+\theta_{2} E\left(w_{t-2} w_{t-1}\right)+\theta_{2} \theta_{1} E\left(w_{t-2} w_{t-2}\right)+\theta_{2}^{2} E\left(w_{t-2} w_{t-3}\right)
\end{aligned}
$$


Collecting terms, then the ACVF for $M A(2)$ at lag 1 is,

$$
\begin{aligned}
\gamma(1) & =\sigma_{t, t-1}+\theta_{1}\left(\sigma_{t, t-2}+\sigma_{t-1, t-1}\right)+\theta_{2}\left(\sigma_{t, t-3}+\sigma_{t-2, t-1}\right) \\
& +\theta_{1}^{2} \sigma_{t-1, t-2}+\theta_{2}^{2} \sigma_{t-2, t-3}+\theta_{1} \theta_{2}\left(\sigma_{t-1, t-3}+\sigma_{t-2, t-2}\right)
\end{aligned}
$$

Similarly, the ACVF for $M A(2)$ at $\operatorname{lag} 2$ is

$$
\begin{aligned}
\gamma(2) & =E\left(w_{t} w_{t-2}\right)+\theta_{1} E\left(w_{t} w_{t-3}\right)+E\left(\theta_{2} w_{t} w_{t-4}\right)+E\left(\theta_{1} w_{t-1} w_{t-2}\right)+\theta_{1}^{2} E\left(w_{t-1} w_{t-3}\right) \\
& +\theta_{1} \theta_{2} E\left(w_{t-1} w_{t-4}\right)+\theta_{2} E\left(w_{t-2} w_{t-2}\right)+\theta_{2} \theta_{1} E\left(w_{t-2} w_{t-3}\right)+\theta_{2}^{2} E\left(w_{t-2} w_{t-4}\right)
\end{aligned}
$$

Collecting terms, then the ACVF for $M A(2)$ at lag 2 is,

$$
\begin{aligned}
\gamma(2) & =\sigma_{t, t-2}+\theta_{1}\left(\sigma_{t, t-3}+\sigma_{t-1, t-2}\right)+\theta_{2}\left(\sigma_{t, t-4}+\sigma_{t-2, t-2}\right) \\
& +\theta_{1}^{2} \sigma_{t-1, t-3}+\theta_{2}^{2} \sigma_{t-2, t-4}+\theta_{1} \theta_{2} \sigma_{t-1, t-4}+\theta_{2} \theta_{1} \sigma_{t-2, t-3}
\end{aligned}
$$

Similarly, $\gamma(h)=0$ for $h \geq 3$.

Dividing (2.15) and (2.16), respectively, by (2.14), yield (2.13), and that completes the proof.

\section{Heteroscedastic Autocorrelation Function (HACF)}

Heteroscedasticity exists if the diagonal elements of $\Sigma$ in (2.4) are not all identical and the disturbance term is free from autocorrelation. In other words, the disturbances are pairwise uncorrelated. This assumption is likely to be realistic one when using crosssectional data. In this case $\Sigma$ can be written as a diagonal matrix with the i-th diagonal element is given by $\sigma_{i i}$. We assume $E\left(w_{t}\right)=0$, and $\operatorname{Cov}\left(w_{i}, w_{j}\right)=\Sigma$, where $\Sigma=\operatorname{diag}\left(\sigma_{11}, \sigma_{22}, \ldots, \sigma_{t t}\right)$. Thus

$$
\Sigma=\left[\begin{array}{cccc}
\sigma_{11} & 0 & \ldots & 0 \\
0 & \sigma_{22} & \ldots & 0 \\
\vdots & \vdots & \ddots & \vdots \\
0 & 0 & \ldots & \sigma_{t t}
\end{array}\right]
$$

Theorem (3.1) derives the HACF, at lag $h$ when $\sigma_{i, j}=0$ for all $i \neq j$, i.e. $\Sigma=\operatorname{diag}\left(\sigma_{11}, \sigma_{22}, \ldots, \sigma_{t t}\right)$ for an $M A(q)$ model. 
Theorem 3.1. Consider the general $M A(q)$ model, $Z_{t}=\sum_{j=0}^{q} \theta_{j} w_{t-j}$, with $E\left(w_{t}\right)=0$ and $\operatorname{Cov}\left(w_{i}, w_{j}\right)=\operatorname{diag}\left(\sigma_{11}, \sigma_{22}, \ldots, \sigma_{t t}\right)$ as given in (3.1). Then the HACF for an $\operatorname{MA}(q)$ at lag $h$ is given by

$$
\rho(h)= \begin{cases}\frac{\sum_{i=0}^{q-h} \theta_{i} \theta_{i+h} \sigma_{t-i-h, t-i-h}}{\sum_{i=0}^{q} \theta_{i}^{2} \sigma_{t-i, t-i}} & , 1 \leq h \leq q \\ 0, & h>q\end{cases}
$$

Proof: Using (2.8) with $h=0$, and $\operatorname{Cov}\left(w_{i}, w_{j}\right)=\operatorname{diag}\left(\sigma_{11}, \sigma_{22}, \ldots, \sigma_{t t}\right)$ as given in (3.1). Then the ACVF for an $M A(q)$ at lag $O$ is

$$
\begin{aligned}
\gamma(0) & =E\left(w_{t} w_{t}\right)+\theta_{1}^{2} E\left(w_{t-1} w_{t-1}\right)+\theta_{2}^{2} E\left(w_{t-2} w_{t-2}\right)+\cdots+\theta_{q}^{2} E\left(w_{t-q} w_{t-q}\right) \\
& =\sigma_{t, t}+\theta_{1}^{2} \sigma_{t-1, t-1}+\theta_{2}^{2} \sigma_{t-2, t-2}+\cdots+\theta_{q}^{2} \sigma_{t-q, t-q}
\end{aligned}
$$

Then the ACVF for an $M A(q)$ at lag 0 , i.e. the variance of an $M A(q)$ model is

$$
\gamma(0)=\sum_{i=0}^{q} \theta_{i}^{2} \sigma_{t-i, t-i}
$$

Using (2.8) with $h=1$, and $\operatorname{Cov}\left(w_{i}, w_{j}\right)=\operatorname{diag}\left(\sigma_{11}, \sigma_{22}, \ldots, \sigma_{t t}\right)$ as given in (3.1). Then the ACVF for an $M A(q)$ at lag 1 is

$$
\begin{aligned}
\gamma(1) & =E\left(\theta_{1} w_{t-1} w_{t-1}\right)+\theta_{2} \theta_{1} E\left(w_{t-2} w_{t-2}\right)+\cdots+\theta_{q} \theta_{q-1} E\left(w_{t-q} w_{t-q}\right) \\
& =\theta_{1} \sigma_{t-1, t-1}+\theta_{2} \theta_{1} \sigma_{t-2, t-2}+\cdots+\theta_{q} \theta_{q-1} \sigma_{t-q, t-q} \\
& =\sum_{i=1}^{q} \theta_{i} \theta_{i-1} \sigma_{t-i, t-i}
\end{aligned}
$$

Then the ACVF for an $M A(q)$ at $\operatorname{lag} 1$ is

$$
\gamma(1)=\sum_{i=0}^{q-1} \theta_{i} \theta_{i+1} \sigma_{t-i-1, t-i-1}
$$

Similarly, the ACVF for an $M A(q)$ at lag $h$ for $h=1,2, \ldots, q$ is

$$
\gamma(h)=\left\{\begin{array}{lc}
\sum_{i=0}^{q-h} \theta_{i} \theta_{i+h} \sigma_{t-i-h, t-i-h}, & 0 \leq h \leq q \\
0, & h>q
\end{array}\right.
$$

Dividing (3.5) by (3.3), yields (3.2), and that completes the proof.

The next corollaries (3.2) and (3.3) derive the HACF at lag $h$ for an $M A(1)$ and $M A(2)$ models. 
Corollary 3.2. Consider $M A(1)$ model, $Z_{t}=w_{t}+\theta w_{t-1}$, with $E\left(w_{t}\right)=0$ and $\operatorname{Cov}\left(w_{i}, w_{j}\right)=\operatorname{diag}\left(\sigma_{11}, \sigma_{22}, \ldots, \sigma_{t t}\right)$ as given in (3.1). Then the HACF for an MA(1) at lag $h$ is given by

$$
\rho(h)= \begin{cases}\frac{\theta \sigma_{t-1, t-1}}{\sigma_{t, t}+\theta^{2} \sigma_{t-1, t-1}}, & h=1 \\ 0, & h \geq 2\end{cases}
$$

Proof: Using (2.8) with $h=0$, and $\operatorname{Cov}\left(w_{i}, w_{j}\right)=\operatorname{diag}\left(\sigma_{11}, \sigma_{22}, \ldots, \sigma_{t t}\right)$ as given in (3.1). Then the ACVF for an $M A(1)$ at lag 0 is

$$
\begin{aligned}
\gamma(0) & =E\left[\left(w_{t}+\theta w_{t-1}\right)\left(w_{t}+\theta w_{t-1}\right)\right] \\
& =E\left(w_{t} w_{t}\right)+\theta E\left(w_{t} w_{t-1}\right)+E\left(\theta w_{t-1} w_{t}\right)+\theta^{2} E\left(w_{t-1} w_{t-1}\right)
\end{aligned}
$$

Then the variance of an $M A(1)$ model is,

$$
\gamma(0)=\sigma_{t, t}+\theta^{2} \sigma_{t-1, t-1}
$$

The ACVF for an $M A(1)$ at lag 1 is

$$
\begin{aligned}
\gamma(1) & =E\left[\left(w_{t}+\theta w_{t-1}\right)\left(w_{t-1}+\theta w_{t-2}\right)\right] \\
& =E\left(w_{t} w_{t-1}\right)+\theta E\left(w_{t} w_{t-2}\right)+E\left(\theta w_{t-1} w_{t-1}\right)+\theta^{2} E\left(w_{t-1} w_{t-2}\right)
\end{aligned}
$$

Collecting terms, then the ACVF for an $M A(1)$ at lag 1 is,

$$
\gamma(1)=\theta \sigma_{t-1, t-1}
$$

Similarly, $\gamma(h)=0$ for $h \geq 2$

Dividing (3.8) by (3.7), yields (3.6), and that completes the proof.

Corollary 3.3. Consider $M A(2)$ model, $Z_{t}=w_{t}+\theta_{1} w_{t-1}+\theta_{2} w_{t-2}$, with $E\left(w_{t}\right)=0$ and $\operatorname{Cov}\left(w_{i}, w_{j}\right)=\operatorname{diag}\left(\sigma_{11}, \sigma_{22}, \ldots, \sigma_{t t}\right)$ as given in (3.1). Then the GHACF for MA(2) at lag $h$ is given by

$$
\begin{aligned}
& \rho(1)=\frac{\theta_{1} \sigma_{t-1, t-1}+\theta_{1} \theta_{2} \sigma_{t-2, t-2}}{\sigma_{t, t}+\theta_{1}^{2} \sigma_{t-1, t-1}+\theta_{2}^{2} \sigma_{t-2, t-2}} \\
& \rho(2)=\frac{\theta_{2} \sigma_{t-2, t-2}}{\sigma_{t, t}+\theta_{1}^{2} \sigma_{t-1, t-1}+\theta_{2}^{2} \sigma_{t-2, t-2}} \\
& \rho(h)=0 \text { for } h \geq 3
\end{aligned}
$$


Proof: Using (2.8) with $h=0$, and $\operatorname{Cov}\left(w_{i}, w_{j}\right)=\operatorname{diag}\left(\sigma_{11}, \sigma_{22}, \ldots, \sigma_{t t}\right)$ as given in (3.1). Then the variance for an $M A(2)$ model,

$$
\gamma(0)=E\left(w_{t} w_{t}\right)+\theta_{1}^{2} E\left(w_{t-1} w_{t-1}\right)+\theta_{2}^{2} E\left(w_{t-2} w_{t-2}\right)
$$

Then,

$$
\gamma(0)=\sigma_{t, t}+\theta_{1}^{2} \sigma_{t-1, t-1}+\theta_{2}^{2} \sigma_{t-2, t-2}
$$

Using (2.8) with $h=1$, and $\operatorname{Cov}\left(w_{i}, w_{j}\right)=\operatorname{diag}\left(\sigma_{11}, \sigma_{22}, \ldots, \sigma_{t t}\right)$ as given in (3.1). Then the ACVF for an $M A(2)$ at lag 1 is

$$
\gamma(1)=\theta_{1} E\left(w_{t-1} w_{t-1}\right)+\theta_{1} \theta_{2} E\left(w_{t-2} w_{t-2}\right)
$$

Collecting terms, then the ACVF for an MA(2) at lag 1 is,

$$
\gamma(1)=\theta_{1} \sigma_{t-1, t-1}+\theta_{1} \theta_{2} \sigma_{t-2, t-2}
$$

Similarly, the ACVF for an $M A(2)$ at lag 2 is

$$
\gamma(2)=\theta_{2} E\left(w_{t-2} w_{t-2}\right)
$$

Collecting terms, then the ACVF for $M A(2)$ at lag 2 is,

$$
\gamma(2)=\theta_{2} \sigma_{t-2, t-2}
$$

Similarly, $\gamma(h)=0$ for $h \geq 3$.

Dividing (3.11) and (3.12), respectively, by (3.10), yield (3.9), and that completes the proof.

Special Cases: Homoscedasticity exists if the diagonal elements of $\Sigma$ in (2.4) are all identical and the disturbance term, $w$, is free from autocorrelation, i.e. $\sigma_{i j}=0 \forall i \neq j$. In this case, the disturbance term is a sequence of independent, identically distributed random variables.

The next Corollaries (3.4) through (3.6) show the ACF for MA models by using Theorem (3.1) and Corollaries (3.2) and (3.3).

Corollary 3.4. Consider the general $M A(q)$ model, $Z_{t}=\sum_{j=0}^{q} \theta_{j} w_{t-j}$, with $E\left(w_{t}\right)=0$ and $\operatorname{Cov}\left(w_{i}, w_{j}\right)=\operatorname{Var}\left(w_{t}\right)=\sigma^{2} \forall t$. By setting $\sigma_{t-i, t-i}=\sigma_{t-i-h, t-i-h}=\sigma^{2}$ in (3.2). Then the ACF for an $M A(q)$ at lag $h$ is given by

$$
\rho(h)=\frac{\sum_{i=0}^{q-h} \theta_{i} \theta_{i+h}}{\sum_{i=0}^{q} \theta_{i}^{2}} \text { for } 1 \leq h \leq q \text { and } \rho(h)=0 \text { for } h>q,
$$

which is the well known ACF for an MA(q) model. 
Corollary 3.5: Consider $M A(1)$ model, $Z_{t}=w_{t}+\theta w_{t-1}$, with $E\left(w_{t}\right)=0$ and $\operatorname{Cov}\left(w_{i}, w_{j}\right)=\operatorname{Var}\left(w_{t}\right)=\sigma^{2} \forall t$. By setting $\sigma_{t, t}=\sigma_{t-1, t-1}=\sigma^{2}$ in (3.6). Then the ACF for an $M A(1)$ at lag $h$ is given by

$$
\rho(h)=\frac{\theta}{1+\theta^{2}} \text { for } h=1 \text { and } \rho(h)=0 \text { for } h \geq 2,
$$

which is the well known ACF for an MA(1) model.

Corollary 3.6. Consider $M A(2)$ model, $Z_{t}=w_{t}+\theta_{1} w_{t-1}+\theta_{2} w_{t-2}$, with $E\left(w_{t}\right)=0$ and $\operatorname{Cov}\left(w_{i}, w_{j}\right)=\operatorname{Var}\left(w_{t}\right)=\sigma^{2} \forall t$. By setting $\quad \sigma_{t, t}=\sigma_{t-1, t-1}=\sigma_{t-2, t-2}=\sigma^{2}$ in (3.9). Then the ACF for an MA(2) at lag $h$ is given by

$$
\rho(1)=\frac{\theta_{1}\left(1+\theta_{2}\right)}{1+\theta_{1}^{2}+\theta_{2}^{2}}, \rho(2)=\frac{\theta_{2}}{1+\theta_{1}^{2}+\theta_{2}^{2}}, \rho(h)=0 \text { for } h \geq 3,
$$

which is the well known ACF for an MA(2) model.

\section{Summary and Future Research}

This paper has investigated an important statistical problem concerning the autocorrelation function, ACF, in the presence of heteroscedasticity disturbances in $q$-th order moving, $M A(q)$, models. We have derived explicit equations for GHACF for an $M A(q)$ when the disturbance terms follow the general structure covariance matrixstructure, $\Sigma$, and when $\Sigma=\operatorname{diag}\left(\sigma_{11}, \sigma_{22}, \ldots, \sigma_{\mathrm{tt}}\right)$. The forms of the explicit equations depend essentially on the moving average coefficients and covariance structure of the disturbance terms.

The plan of the future research is to extend the explicit equations that we have derived in this paper for ACF in the presence of heteroscedasticity disturbances in the general form of the autoregressive moving average models with orders $p$ and $q, A R M A,(p, q)$.

\section{References}

1. Bera, A., Bubnys, E., and Park, H. (2005). "Conditional Heteroscedasticity in the Market Model and Efficient Estimates of Betas," Financial Review, Vol. 23, 2, 201-214.

2. Bumb, B. and Kelejian, H. (1983). "Autocorrelated and Heteroscedastic Disturbances in Linear Regression Analysis: A Monte Carlo study," The Indian Journal of Statistics, 45, Series B, Pt. 2, 257--270.

3. Demos, A. (2000). "The Autocorrelation Function of Conditionally Heteroskedastic in Mean Models. Athens University of Economics and Business, Department of International and European Economic Studies.

4. Dong, L., Shiqing L., and Howell T. (2012). On moving-average models with feedback. Bernoulli 18(2), 2012, 735-745. 
5. Jonathan D. Cryer and Kung-Sik Chan (2008). Time Series Analysis with Applications in $R$, Second Edition, Springer.

6. Praetz, P. (2008). "A Note on the Effect of Autocorrelation on Multiple Regression Statistics," Australian and New Zealand Journal of Statistics, 23(3), 309-313.

7. Safi S. (2009). "Explicit Equations for ACF in the Presence of Heteroscedasticity Disturbances in First-Order Autoregressive Models, AR(1)". The Journal of the Islamic University of Gaza, Vol. 17, No. 2, 97-107.

8. Safi, S. (2011). Explicit Equations for ACF in Autoregressive Processes in the Presence of Heteroscedasticity Disturbances. The Journal of Modern Applied Statistical Methods, 10(2), 625-631.

9. Stockhammar, P. (2010). "Some Contributions to Filtering, Modeling and Forecasting of Heteroscedastic Time Series" Doctoral Dissertation, Department of Statistics, Stockholm University, S-106 91 Stockholm, Sweden.

10. Studenmund, A.H. (2011). "Using Econometrics: A Practical Guide" Prentice Hall, 3rd ed. New Jersey, USA.

11. Wallentin, B. and Agren, A. (2002). "Test of heteroscedasticity in a regression model in the presence of measurement errors," Economics Letters, 76, 205-211. 